\title{
An ERG Gene Analysis in Two Cases with Metastatic Castration-resistant Prostate Cancer in Which Abiraterone Demonstrated Long-term Efficacy
}

\author{
Tsuyoshi Yoshizawa ${ }^{1}$, Kenya Yamaguchi ${ }^{1}$, Nozomu Kawata ${ }^{2}$, Hitomi Ryuzaki ${ }^{3}$, \\ Masahiro Ogawa ${ }^{3}$, Daisuke Obinata ${ }^{1}$, Junichi Mochida ${ }^{1}$ and Satoru Takahashi ${ }^{1}$
}

\begin{abstract}
:
We analyzed the ERG gene status using fluorescence in situ hybridization (FISH) in two chemotherapynaïve cases with metastatic castration-resistant prostate cancer (mCRPC) in which abiraterone demonstrated a long-term durable complete response. FISH identified Class 1 Edel and Class 2+ Edel in case 1, and Class 1 Edel in case 2. Our experience suggests that abiraterone may be effective in cases with mCRPC and ERG gene abnormalities, particularly Class 2+ Edel or Class 1 Edel. This is the first report of two cases with mCRPC that simultaneously investigated the ERG gene status and clinical aspects, including image evaluations and pathology.
\end{abstract}

Key words: ERG gene, fluorescence in situ hybridization, metastatic castration-resistant prostate cancer, abiraterone, durable complete response

(Intern Med 59: 395-399, 2020)

(DOI: 10.2169/internalmedicine.3302-19)

\section{Introduction}

Abiraterone suppresses androgen synthesis by selectively inhibiting CYP17A, a metabolic enzyme involved in the synthesis of androgen from cholesterol. The addition of abiraterone reduces androgen concentrations in the blood and tissues and exhibits a therapeutic effect that is superior to that of androgen-deprivation therapy (ADT) using a luteinizing hormone-releasing hormone analogue (LHRHa) alone (1).

According to the interim analysis results of the COU-AA302 trial on 1,088 pre-chemotherapy patients with castration-resistant prostate cancer (CRPC), the abiraterone group showed a significantly longer progression-free survival according to the imaging data (2) and significant improvements in pain and the quality of life (3) compared to the placebo group. The final analysis results included a median overall survival of 34.7 months in the abiraterone group and 30.3 months in the placebo group (hazard ratio: 0.81; $\mathrm{p}=0.0033$ ) (4). The use of abiraterone, enzalutamide and docetaxel for metastatic CRPC (mCRPC) has both a high evidence level and recommendation grade; therefore, such regimens are considered established therapies (5). However, a consensus on the timing and sequence of using these drugs has not been reached.

Few biomarkers have proven useful for drug selection. A recent sub-analysis of the COU-AA-302 trial suggested that ERG gene rearrangement in archival sample could function as a predictive biomarker for patients with mCRPC treated with abiraterone (6). However, patients with liver metastasis were excluded from the COU-AA-302 trial. Therefore, the association between the ERG gene status in primary prostate tumor and sensitivity to abiraterone treatment in mCRPC patients with liver metastasis still remains unknown.

In the present study, we evaluated the ERG gene rearrangement in two cases of mCRPC with liver and bone-only metastasis in which abiraterone demonstrated long-term efficacy.

${ }^{1}$ Department of Urology, Nihon University School of Medicine, Japan, ${ }^{2}$ Department of Urology, Nihon University Hospital, Japan and ${ }^{3}$ Division of Gastroenterology and Hepatology, Department of Medicine, Nihon University Hospital, Japan

Received: May 7, 2019; Accepted: September 3, 2019; Advance Publication by J-STAGE: October 17, 2019

Correspondence to Dr. Satoru Takahashi, takahashi.satoru@nihon-u.ac.jp 

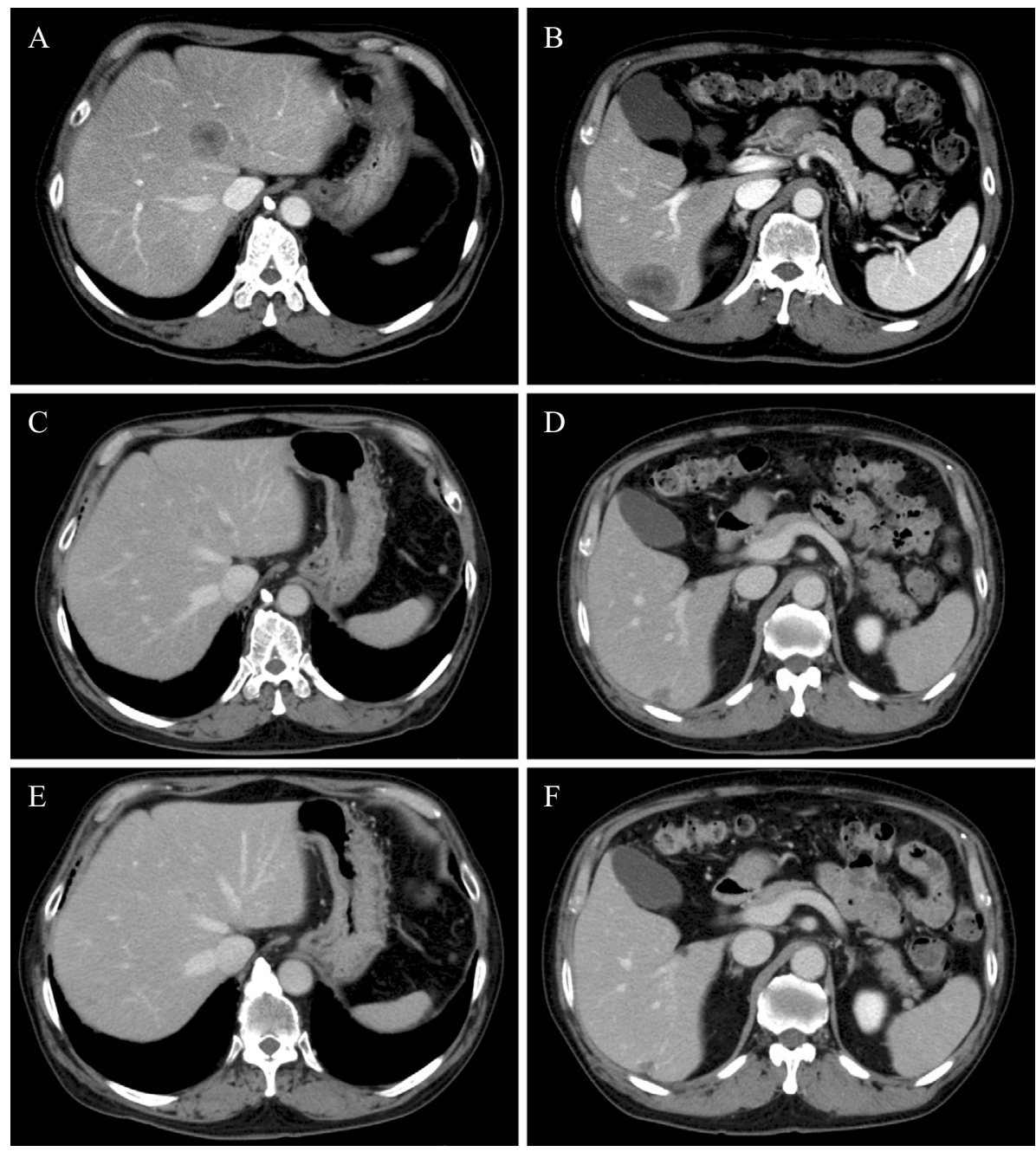

Figure 1. Time course of contrast-enhanced computed tomography (CECT) images in case 1. (A) In S4 of the liver, a hypovascular tumor was found with 30-mm, faint, ring-shaped enhancement (before abiraterone treatment). (B) In S6 of the liver, a hypovascular tumor was found with 45-mm, faint, ring-shaped enhancement (before abiraterone treatment). (C) The S4 tumor shrank to $7 \mathrm{~mm}$, which indicated a partial response (PR) (6 months after abiraterone administration). (D) The S6 tumor shrank to $10 \mathrm{~mm}$, which indicated a PR. A scar-shaped shadow is noted (6 months after abiraterone administration). (E) The $\mathrm{S} 4$ tumor achieved a complete response (CR) (12 months after abiraterone administration). (F) The S6 tumor achieved a CR. A scar-shaped shadow is noted (12 months after abiraterone administration).

\section{Case Reports}

Case 1 involved a 78-year-old patient with a performance status (PS) of 0 and history of hypertension. With a prostate-specific antigen (PSA) level of $8.19 \mathrm{ng} / \mathrm{mL}$, he underwent prostate biopsy (18 cores) in May 2009 that detected adenocarcinoma in 5 cores. Since subsequent examinations showed no metastasis, he underwent radical prostatectomy, and the resulting histopathology showed Gleason $4+5=9$ and pT2b.

Following PSA failure, salvage radiation therapy and ADT, he developed CRPC in September 2013. The PSA level at that time was $19.57 \mathrm{ng} / \mathrm{mL}$, and contrast-enhanced computed tomography (CECT) revealed tumors in S4 and S6 of the liver (Fig. 1A, B). A needle biopsy of the S6 tu- mor revealed a PSA-positive metastatic adenocarcinoma. Additional abiraterone was started from September 2014. The period of its PSA efficacy was 18 months (Fig. 2). Six months after abiraterone administration, CECT revealed a partial response (PR) (Fig. 1C , D) and biopsy of S6 tumor did not find viable carcinoma cells. Furthermore, CECT performed 12 months after abiraterone yielded a complete response (CR) (Fig. 1E, F) that lasted for 6 months. Fluorescence in situ hybridization (FISH) of the radical prostatectomy specimen showed Class 1 Edel and Class 2+ Edel (Fig. 3A, B).

Case 2 involved an 80-year-old patient with a PS of 1 , low back pain, and a history of hyperlipidemia. With a PSA level of $11.8 \mathrm{ng} / \mathrm{mL}$, he underwent a prostate biopsy (30 cores) in April 2014 at another hospital, which revealed high-grade prostatic intraepithelial neoplasia (PIN) in one 


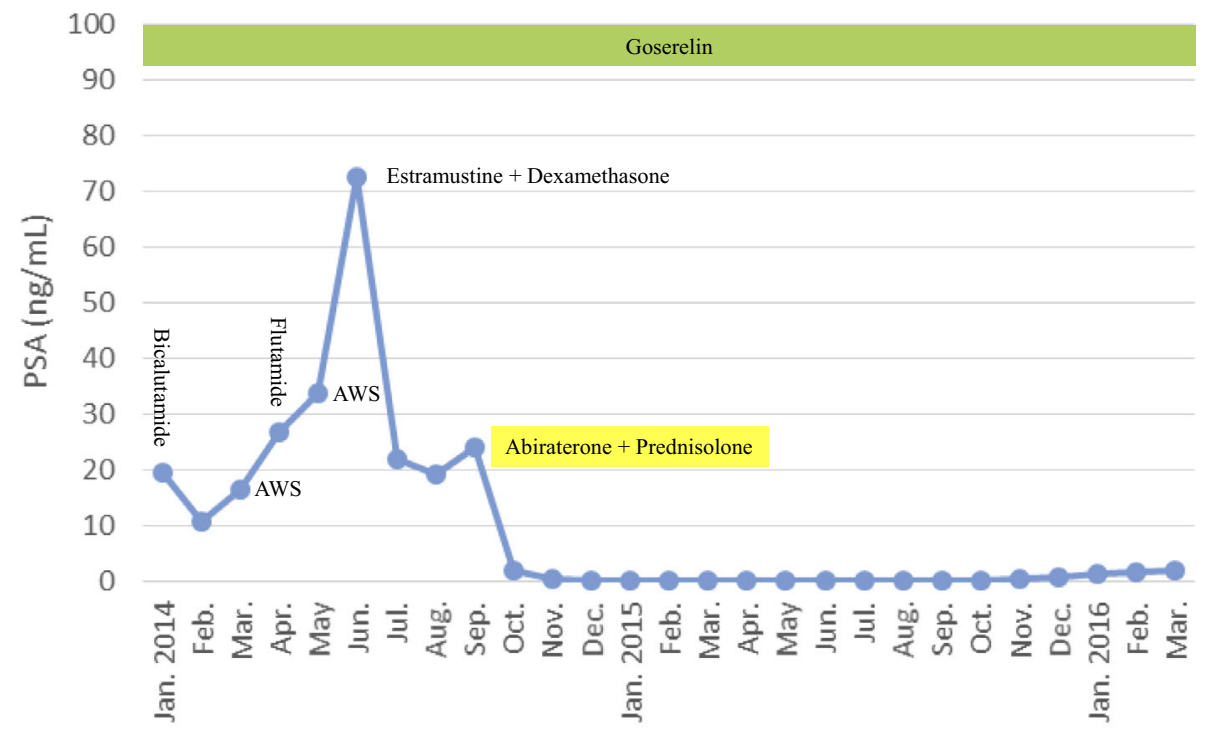

Figure 2. Endocrine therapy and changes in the PSA level in case 1. PSA: prostate-specific antigen, AWS: anti-androgen withdrawal syndrome
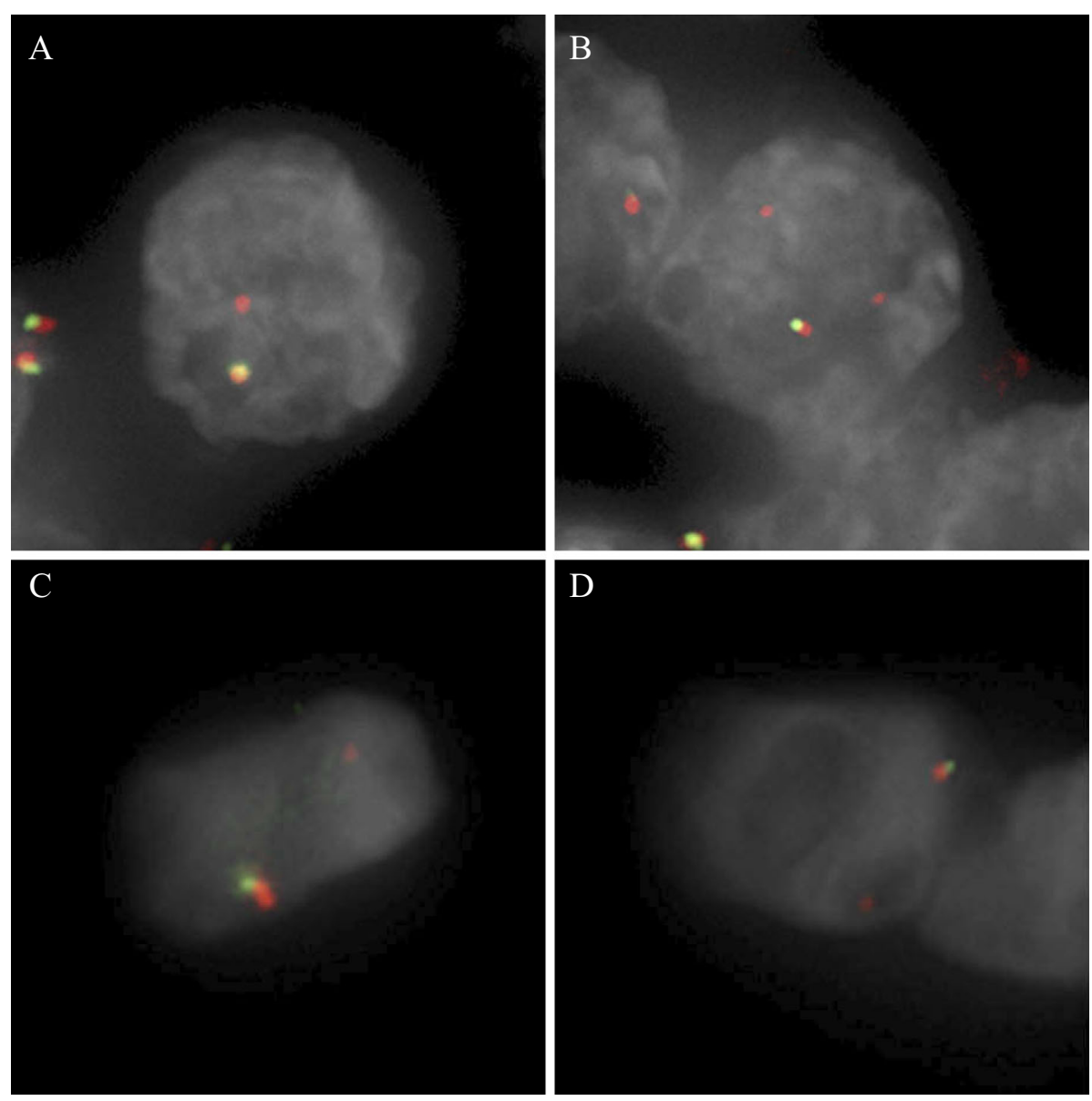

Figure 3. ERG gene rearrangement according to fluorescence in situ hybridization (FISH) (the ERG gene FISH probe signal is red at the centromere end and yellowish green at the telomere end). (A) FISH using radical prostatectomy specimen in case 1. Class 1 Edel was found. (B) FISH using radical prostatectomy specimen in case 1. Class 2+ Edel was found. (C, D) FISH using prostate biopsy tissue in case 2. Class 1 Edel was found. 


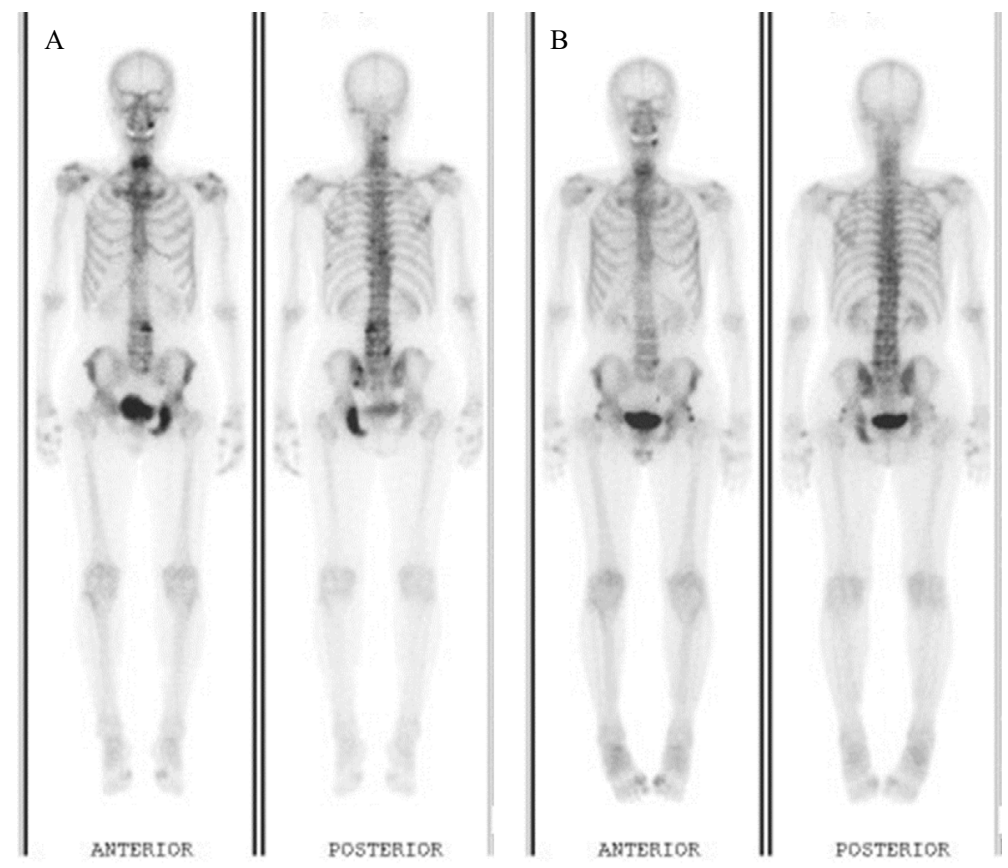

Figure 4. Chronological changes in bone scintigraphy in case 2. (A) Metastases (abnormal masses) in the lumbar vertebrae, left iliac bone, and left ischial bone were found (before abiraterone administration). (B) Metastases (abnormal masses) in the lumbar vertebrae, left iliac bone, and left ischial bone disappeared (12 months after abiraterone administration).

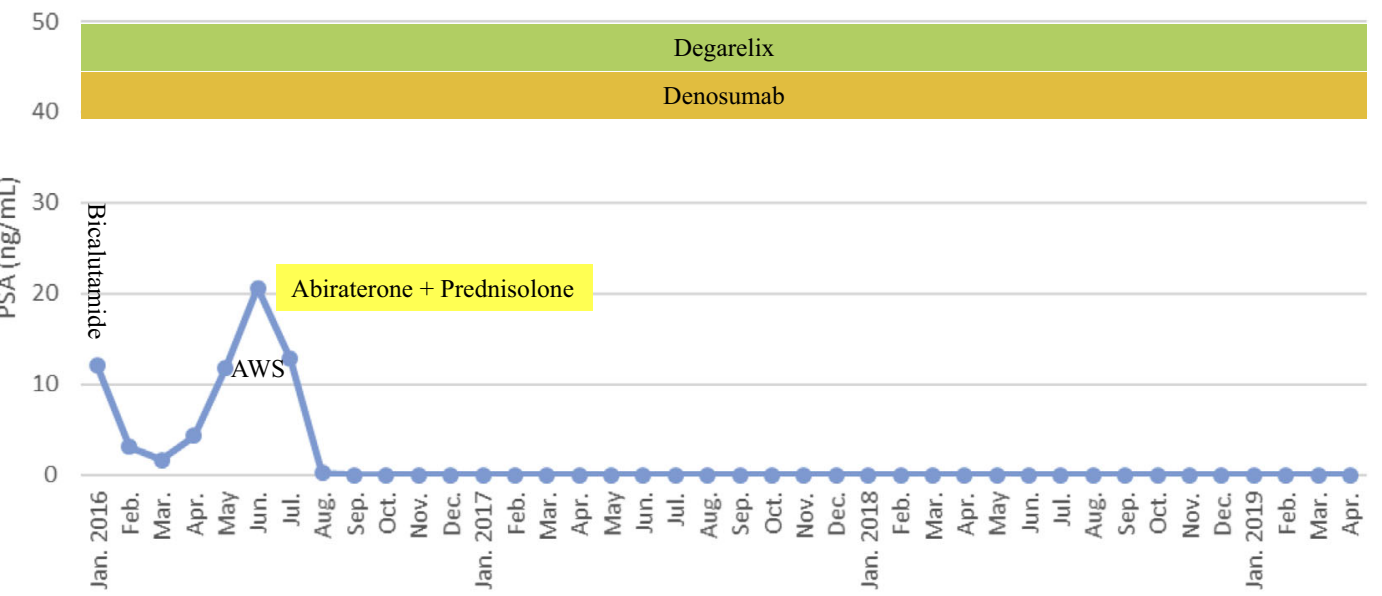

Figure 5. Endocrine therapy and changes in the PSA level in case 2. PSA: prostate-specific antigen, AWS: anti-androgen withdrawal syndrome

2016, when his PSA level was $12.12 \mathrm{ng} / \mathrm{mL}$. Subsequently, he underwent a re-biopsy (14 cores), which showed Gleason $4+4=8$ adenocarcinoma in 5 cores. Bone scintigraphy found metastases in the lumbar vertebrae, left iliac bone, and left ischial bone (Fig. 4A). The addition of abiraterone was started from June 2016, and its duration of PSA efficacy was 33 months (Fig. 5). In bone scintigraphy performed 12 months after abiraterone administration, the metastatic foci had disappeared (Fig. 4B). The metastatic foci currently remain eliminated, and low back pain is also no longer found. FISH of the prostate biopsy tissue showed Class 1 Edel (Fig. 3C, D).

\section{Discussion}

Compared to benign prostate disease, the ERG expression is increased in prostate cancer (7). An increased ERG expression is associated with an increased risk of progression for patients under active surveillance of prostate cancer (8). In addition to PSA levels and the Gleason score at the detection of prostate cancer, TMPRSS2-ERG fusion and Class $2+$ Edel are poor prognostic factors and may be new factors for patient stratification (9).

Attard et al. evaluated the ERG gene status in 445 cases of prostate cancer using FISH, focusing on ERG gene anomalies (9). According to this study, ERG gene rearrange- 
ments were found in $30 \%$ of cases. The ERG gene status is classified as Class N (normal), Class Esplit, Class 1 Edel, and Class 2+ Edel. Class Edel cases have a significantly lower cause-specific and overall survival than Class $\mathrm{N}$ cases. In addition, in cases of Class 2+ Edel rather than Class 1 Edel, the cause-specific and overall survival are reported to be significantly lower than in Class $\mathrm{N}$ cases (9). Furthermore, Attard et al. reported that administering abiraterone to patients with mCRPC tended to delay the time to PSA progression and improve the radiographic progression-free survival in the Class 2+ Edel group compared with a nonrearranged group (6). In case 1 of the present report, FISH showed Class 2+ Edel, which supports the results of the report by Attard et al., despite the liver metastasis. This is the first report to indicate that Class 2+ Edel in archival tissue predicts a good response to abiraterone treatment in mCRPC patients with liver metastasis.

In prostate cancer cells with TMPRSS2-ERG fusion, ERG may enhance intracrine androgen synthesis (androgen synthesis by cancer cells) by inducing the expression of AKR1C3, one of the enzymes responsible for androgen synthesis. Therefore, Powell et al. hypothesized that the suppression of gonadal androgen alone is not sufficient, but the suppression of androgen synthesis by abiraterone still has utility in treatment: because CYP17A is located upstream of AKR1C3, the suppression of CYP17A may also be effective for AKR1C3-dependent cancer (10).

However, in case 2, FISH did not show Class 2+ Edel. Nevertheless, despite having Class 1 Edel, abiraterone had a therapeutic effect over a long-term period. In addition to FISH, next-generation sequencing (NGS) and immunohistochemistry (IHC) are useful for detecting the mutation and amplification of ERG. However, in archival tissue, there are concerns that DNA is cross-linked by formalin and becomes fragmented during processing, resulting in a lack of sequence accuracy. In addition, IHC lacks reliability regarding the expression level of ERG. In contrast, FISH demonstrates a high degree of reliability in identifying mutations and an amplification of ERG. Therefore, we used FISH to analyze the ERG status in the present study.

When performing FISH using prostate biopsy tissue specimens, very few sites show Class Edel (11). Therefore, Class 2+ Edel may be identified in other cancer tissue specimens. Even with FISH, the evaluation of the ERG gene status using prostate biopsy tissue specimens is limited (12).

However, a previous report suggested that Class 1 Edel and Class 2+ Edel coexist in about half of patients (13), so abiraterone might still show efficacy in cases of Class 1 Edel. Furthermore, a previous study suggested that 21q22 (ERG gene fusion) is observed after performing therapeutic intervention (7). This means that the ERG gene status in CRPC patients may change over the course of treatment. Therefore, undetectable Class 2+ Edel may exist in cases with Class 1 Edel. Abiraterone may be effective in patients with ERG gene abnormalities, even if organ metastases are found.

The authors state that they have no Conflict of Interest (COI).

\section{Acknowledgement}

We are thankful to Masahisa Tsuji who assisted us with the FISH analysis.

\section{References}

1. Taplin ME, Montgomery B, Logothetis CJ, et al. Intense androgen-deprivation therapy with abiraterone acetate plus leuprolide acetate in patients with localized high-risk prostate cancer: results of a randomized phase II neoadjuvant study. J Clin Oncol 32: 3705-3715, 2014.

2. Ryan CJ, Smith MR, de Bono JS, et al. Abiraterone in metastatic prostate cancer without previous chemotherapy. N Engl J Med 368: 138-148, 2013.

3. Basch E, Autio K, Ryan CJ, et al. Abiraterone acetate plus prednisone versus prednisone alone in chemotherapy-naive men with metastatic castration-resistant prostate cancer: patient-reported outcome results of a randomised phase 3 trial. Lancet Oncol 14: 1193-1199, 2013.

4. Ryan CJ, Smith MR, Fizazi K, et al. Abiraterone acetate plus prednisone versus placebo plus prednisone in chemotherapy-naive men with metastatic castration-resistant prostate cancer (COU-AA302): final overall survival analysis of a randomised, double-blind, placebo-controlled phase 3 study. Lancet Oncol 16: 152-160, 2015.

5. Cornford P, Bellmunt J, Bolla M, et al. EAU-ESTRO-SIOG guidelines on prostate cancer. Part II: treatment of relapsing, metastatic, and castration-resistant prostate cancer. Eur Urol 71: 630-642, 2017.

6. Attard G, de Bono JS, Logothetis CJ, et al. Improvements in radiographic progression-free survival stratified by ERG gene status in metastatic castration-resistant prostate cancer patients treated with abiraterone acetate. Clin Cancer Res 21: 1621-1627, 2015.

7. Tomlins SA, Rhodes DR, Perner S, et al. Recurrent fusion of TMPRSS2 and ETS transcription factor genes in prostate cancer. Science 310: 644-648, 2005.

8. Berg KD, Vainer B, Thomsen FB, et al. ERG protein expression in diagnostic specimens is associated with increased risk of progression during active surveillance for prostate cancer. Eur Urol 66: 851-860, 2014.

9. Attard G, Clark J, Ambroisine L, et al. Duplication of the fusion of TMPRSS2 to ERG sequences identifies fatal human prostate cancer. Oncogene 27: 253-263, 2008.

10. Powell K, Semaan L, Conley-LaComb MK, et al. ERG/AKR1C3/ AR constitutes a feed-forward loop for AR signaling in prostate cancer cells. Clin Cancer Res 21: 2569-2579, 2015.

11. Clark J, Attard G, Jhavar S, et al. Complex patterns of ETS gene alteration arise during cancer development in the human prostate. Oncogene 27: 1993-2003, 2008.

12. Brandi F, Grupp K, Hube-Magg C, et al. High concordance of TMPRSS-ERG fusion between primary prostate cancer and its lymph node metastases. Oncol Lett 16: 6238-6244, 2018.

13. Attard G, Swennenhuis JF, Olmos D, et al. Characterization of ERG, AR and PTEN gene status in circulating tumor cells from patients with castration-resistant prostate cancer. Cancer Res 69: 2912-2918, 2009.

The Internal Medicine is an Open Access journal distributed under the Creative Commons Attribution-NonCommercial-NoDerivatives 4.0 International License. To view the details of this license, please visit (https://creativecommons.org/licenses/ by-nc-nd/4.0/).

(C) 2020 The Japanese Society of Internal Medicine Intern Med 59: 395-399, 2020 